11

\title{
Causes and pathophysiology of hypoparathyroidism
}

\author{
Luisella Cianferotti, MD, PhD, Assistant Professor of \\ Endocrinology *, \\ Gemma Marcucci, MD, PhD, Assistant Professor of \\ Endocrinology, \\ Maria Luisa Brandi, MD, PhD, Professor of Endocrinology
}

Department of Surgery and Translational Medicine, University of Florence, Bone Metabolic Diseases Unit, University Hospital of Florence, Italy

\section{A R T I C L E I N F O}

\section{Article history:}

Available online 29 July 2018

\section{Keywords:}

autoimmune hypoparathyroidism syndromic hypoparathyroidism isolated hypoparathyroidism post-surgical hypoparathyroidism chronic hypoparathyroidism autosomal dominant hypocalcemia
Hypoparathyroidism, a disorder characterized by hypocalcemia ensuing from inadequate parathyroid hormone secretion, is a rather rare disorder caused by multiple etiologies. When not caused by inadvertent damage or removal of the parathyroids during neck surgery, it is usually genetically determined. Epidemiological figures of this disease are still scarce and mainly limited to countries where non-anonymous databases are available and to surgical case series. Both the surgical and non-surgical forms pose diagnostic challenges. For surgical hypoparathyroidism, transient forms have to be ruled out even in the long term, in order to avoid unnecessary chronic replacement therapy with calcium and calcitriol. Regarding non-surgical hypoparathyroidism, once referred to as idiopathic, a systematic clinically and genetically-driven approach to define the precise diagnosis have to be pursued. In the case of syndromic hypoparathyroidism, patients have to be screened for associated abnormalities. Autoimmune, non-genetic hypoparathyroidism is still a diagnosis of exclusion, since no specific autoantibodies are specific for this condition.

(C) 2018 Elsevier Ltd. All rights reserved.

\footnotetext{
* Corresponding author. Department of Surgery and Translational Medicine, University of Florence, Bone Metabolic Diseases Unit, University Hospital of Florence, Viale Pieraccini, 6, 50139 Florence, Italy. Fax: +39 0557946303.

E-mail addresses: luisella.cianferotti@unifi.it (L. Cianferotti), gemma.marcucci@unifi.it (G. Marcucci), marialuisa.brandi@ unifi.it (M.L. Brandi).
} 


\section{Introduction (definition and pathophysiology of hypoparathyroidism)}

The term hypoparathyroidism encompasses a broad series of disorders of mineral metabolism of different etiologies, all characterized by inadequate or insufficient parathyroid hormone (PTH) secretion leading to hypocalcemia, hyperphosphatemia and related signs and symptoms [1-4].

PTH is the main regulator of calcium homeostasis and, together with fibroblast growth factor 23 (FGF23), of phosphate homeostasis. The most important consequences of the missing or abnormal parathyroid function can be ascribed to the lack of direct or indirect PTH actions on main organs devoted to the maintenance of calcium homeostasis such as the kidneys, bone and intestine. These organs express the PTH 1 receptor (PTH1R), a G-protein coupled receptor transducing PTH and PTH related peptide (PTHrP) effects on target cells [5]. The interaction of PTH on PTH1R on renal proximal tubular cells enhances the hydroxylation of 25 hydroxyvitamin D [25(OH)D], thus producing 1,25 dihydroxy vitamin $\mathrm{D}\left[1,25(\mathrm{OH})_{2} \mathrm{D}\right]$, i.e. calcitriol, the biologically active hormone responsible of active calcium and phosphate absorption from the intestinal lumen. Parathyroid function is mainly regulated by extracellular calcium ions $\left(\left[\mathrm{Ca}^{2+}\right]\right)$, which interact with the calcium sensing receptor (CaSR), another G-protein coupled receptor primarily expressed on the parathyroid cell membrane and on renal tubular cells [6]. CaSR is a key regulator of calcium homeostasis. When $\left[\mathrm{Ca}^{2+}\right]$ transiently increases the receptor is activated and transcription of PTH gene, translation into mature protein and secretion are inhibited [6]. The relationship between secreted PTH and extracellular calcium ([Ca $\left.\left.{ }^{2+}\right]\right)$, expressed by a sigmoidal curve, is maintained although shifted towards left or right even in the case of PTH insufficiency or excess, respectively, thanks to the expression of at least one copy of the CaSR gene [6]. At the renal tubular level, CaSR is involved in regulating calcium reabsorption.

In the case of PTH inadequacy, intestinal calcium and phosphate absorption decrease. At the kidney level, the tubular transport of calcium (TmCa) is decreased and the tubular transport of phosphate $(\mathrm{TmP})$ is increased, so that individuals with hypoparathyroidism are also prone to hypercalciuria and hyperphosphatemia in addition to the main feature, i.e. hypocalcemia [7]. Hypocalcemia is the main responsible for the development of signs and symptoms related to muscular hyperexcitability, while long-standing hyperphosphatemia leads to the occurrence of soft tissue calcifications (i.e. basal ganglia calcifications, subcapsular cataract). The tendency towards hypercalciuria, which gets worse under calcium and active vitamin D replacement therapy, increase the risk of nephrolithiasis, in particular under calcium and calcitriol replacement therapy. Besides the cohort of sign and symptoms directly related to the disturbances in mineral homeostasis, signs and symptoms due to the lack of PTH action on various tissues and organs such as bone expressing the PTH1R may be present [1].

The products of key developmental genes drive the patterning and migration of the parathyroids, which arise from the third and fourth pharyngeal pouches endoderm with neural crest contribution [8]. Since these developmental structures give rise to multiple organs other than the parathyroids, alterations in these genes are responsible of complex syndromes, in which hypoparathyroidism is usually associated with other clinical manifestations [9]. Conversely, mutations in genes encoding for proteins active at later stages of parathyroid development will give rise to isolated hypoparathyroidism [9]. Syndromic or isolated hypoparathyroidism are usually also referred to as genetic or congenital hypoparathyroidism, despite the fact that they can manifest only in adult life.

Removal, anatomic or vascular damage to the parathyroids during neck surgery (mainly thyroidectomy or parathyroidectomy) represents the main cause of acquired hypoparathyroidism, i.e. postsurgical hypoparathyroidism [10]. Immune-mediated damage of the parathyroids is another cause of acquired hypoparathyroidism, and is usually associated with other autoimmune disorders [11].

Divalent ions, such as magnesium $\left[\mathrm{Mg}^{2+}\right]$, are important both in PTH secretion and action and in muscle excitability. Hypomagnesemia can blunt PTH secretion, may cause peripheral end-organ resistance to PTH and increase neuromuscular excitability per se, so causing a functional, reversible hypoparathyroidism or exacerbating a pre-existing hypoparathyroidism related symptoms [12]. Hypoparathyroidism as a consequence of infiltrative diseases or external beam radiation is a very rare event.

Within the different causes, acquired hypoparathyroidism is the most frequent form, and, within this, postsurgical hypoparathyroidism is the most frequent one, followed by autoimmune hypoparathyroidism [9]. It is estimated that the proportion between postsurgical forms versus non-surgical 
forms is around 3:1 $[9,13]$. This proportion is expected to gradually change over the next years in favor of non-surgical forms. This is due first of all to the increased expertise in endocrine neck surgery and the increased number of endocrine surgery referral centers leading to diminished risk of causing surgical hypoparathyroidism. In addition, because of the improved clinical expertise in diagnosing non-surgical forms of hypoparathyroidism, the increased knowledge of parathyroid development, differentiation and function with the possibility to apply specific genetic tests, the number of identification and proper diagnosis of non surgery-related hypoparathyroidism, once defined as idiopathic, has gradually increased. Still, proper genetic diagnostic procedures related to non-surgical hypoparathyroidism are like to be carried out in endocrine referral centers.

\section{Epidemiology of hypoparathyroidism}

Data on the prevalence and incidence of hypoparathyroidism as a whole and of the different subtypes are still scarce in a number of settings. Epidemiological data on the disease have been carried out mainly in countries where non-anonymous disease registries are available, such as USA and Denmark [14-16]. Recently, in Italy epidemiological studies have been carried out analyzing anonymous health registries through ICD codes, hospital discharge codes and prescription of chronic therapy with active vitamin D [17,18]. No such reports are still available in Asia, South America, Africa, Australia.

Overall, hypoparathyroidism is overall considered a rare disease (i.e. affecting fewer than 1 in 1000-200,000, according to various international definitions), in particular as long as the genetically determined, congenital conditions are considered. Nonetheless, available epidemiological reports reflect mainly the epidemiology of postsurgical hypoparathyroidism, since still the majority of adult cases represent a consequence of neck surgery. In children non-surgical, genetic causes are more common [19].

In the USA, the prevalence of hypoparathyroidism, as estimated by the analysis of a large insurance claims database over a one-year period, was 77,000 cases in a population of approximately 300 million individuals (i.e. 25 cases/100,000 adults-year) [14]. Analogous figures emerged from a smaller longitudinal study performed in Rochester, Minnesota (USA), in which the prevalence of chronic hypoparathyroidism was 37 cases/100,000. Similar studies carried out in Denmark, where non-anonymous regional and national health registries are available, has shown a comparable overall prevalence of hypoparathyroidism of 25.4 cases/100,000 individuals [15]. The prevalence in other European countries such as Norway or Italy, as calculated by the analysis of hospital discharge ICD codes, has appeared to be lower (i.e. 5-10 cases/100,000) [17,21]. This may be due to the methodology by which diagnosis of hypoparathyroidism are retrieved. Indeed, examining just hospital-based registries can be too restrictive and harbor a possible selection bias [20]. Recently, the indirect assessment of cases of hypoparathyroidism through the analysis of prescription databases belonging to an Italian region, with detection of subjects on chronic (at least 6 month-long) therapy with active vitamin D metabolites, has demonstrated a prevalence of 27 cases/100,000 [18], which is in line with previous results in other countries.

The estimate of cases with hypoparathyroidism and their characteristics is essential in order to select cases refractory to conventional therapy with calcium and active vitamin D suitable for therapy with the full-length PTH, which has become available in USA and Europe.

\section{Causes and classification of hypoparathyroidism}

The various forms of hypoparathyroidism can be classified in different ways, i.e. congenital (i.e. manifest at birth) or acquired (i.e. manifest later in life), genetic (if a specific gene alteration is identified) or non-genetic, isolated or syndromic (i.e. associated with non-parathyroid related dysmorphisms/disturbances) disease, on the basis of clinical features and etiology (Fig. 1). A specific form of hypoparathyroidism, i.e. autoimmune hypoparathyroidism, usually classified as acquired, can be isolated or syndromic if associated with other (usually autoimmune) disorders, genetic or non-genetic, depending on the presence or absence of specific mutation, as detailed later in this paper [4,9]. Genetic hypoparathyroidism is usually referred also to as congenital hypoparathyroidism, even if the clinical manifestation of the calcium disturbance is not present at birth but becomes manifest later in life, 


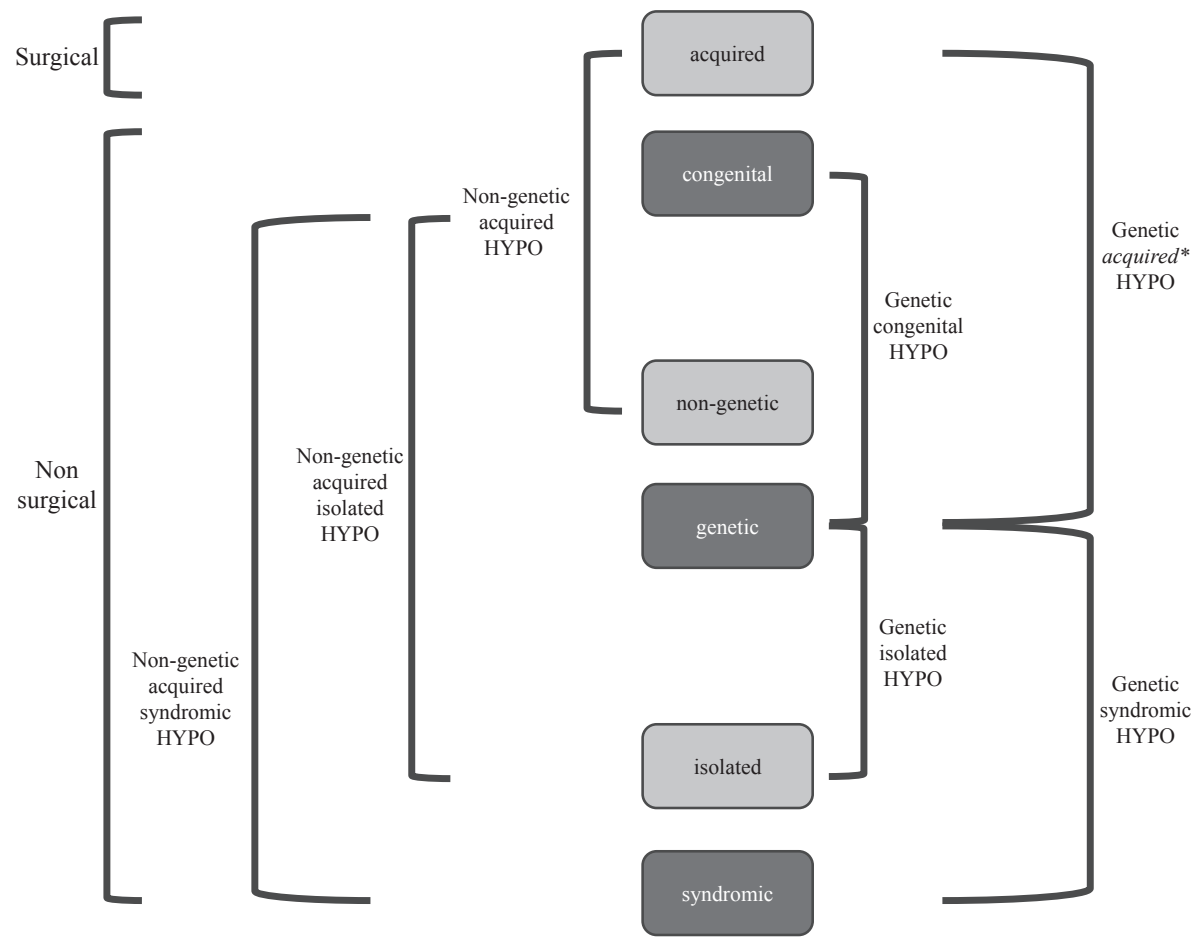

Fig. 1. Classification of hypoparathyroid disorders according to different modalities. Hypoparathyroidism (HYPO) can be classified according to the time of onset (congenital or acquired), presence or absence of a genetic cause (genetic and non genetic), association with other non-parathyroid related clinical manifestations (isolated or syndromic). Acquired hypoparathyroidism is mainly nongenetic and can be isolated or syndromic such as in non-monogenic autoimmune polyendocrinopathies. Genetic hypoparathyroidism, isolated or syndromic, can be congenital or inappropriately defined as acquired* in the case is not manifest at birth or neonatally but later in life. Surgical hypoparathyroidism is an acquired form of hypoparathyroidism. Using this latter way to classify, all the other forms of hypoarpathyroidism can be defined as non surgical.

especially in the cases in which a residual PTH secretion can be demonstrated [9]. With this respect, some genetic/hereditary forms of hypoparathyroidism with reduced penetrance and expressivity in terms of clinical manifestations may be inappropriately defined as acquired (Fig. 1). Considering that most cases of hypoparathyroidism ensue after neck surgery, hypoparathyroidism can be also classified as post-surgical and non-surgical, with the latter one broadly including all the forms of different origin (once inappropriately named as idiopathic).

\section{Genetic hypoparathyroidism}

Genetic or monogenic hypoparathyroidism may ensue from developmental defects usually involving multiple organs mainly arising from the third and fourth pharyngeal pouches, abnormalities in genes encoding for key proteins specific for parathyroid development and function, mutations in genes active early in life in modulating tolerance mechanisms important for adaptive immunity and causing non-surgical damage to the parathyroids later in life, mitochondrial disorders and other rare causes (Table 1) [4,9]. These disorders can originate from de novo or inherited mutations or chromosomal rearrangements, occurring in germ cells (de novo or inherited germline mutations) or at early stages of zygote development (post-zygotic somatic mutations), determining genetic mosaicism in the adult organism. In general, as previously stated, mutations occurring early in development will cause broader defects involving multiple organs (syndromic hypoparathyroidism), while more specific 
Table 1

Classification of genetic hypoparathyroidism according to the origin of the defect: genetically-determined abnormality in development of multiple organs, genetically-determined abnormalities in genes encoding for key proteins for parathyroid development and function, immune system abnormalities, mitochondrial diseases, and other disorders). If a specific gene mutations (indicated in italic; "del." = chromosomal deletion) is responsible for the disease, it is shown in the right column (see text for gene full name, chromosomal location, pattern of inheritance, OMIM classification).

\begin{tabular}{ll}
\hline Disorder & $\begin{array}{l}\text { Cytogenetic/genetic } \\
\text { alteration }\end{array}$ \\
\hline Developmental abnormalities & del. 22q11.2 (TBX1) \\
- DiGeorge syndrome type 1 (DGS1) & del. 10p14-p13 (NEBL) \\
- DiGeorge syndrome type 2 (DGS2) & GHD7 \\
- CHARGE syndrome & TBCE \\
- Hypoparathyroidism, sensorineural deafness and renal dysplasia (HDR) & TBCE \\
- Hypoparathyroidism, retardation, and dysmorphism syndrome (HRD) & FAM111A \\
- Kenny-Caffey syndrome type 1 (KCS1) & FAM111A \\
- Kenny-Caffey syndrome type 2 (KCS2) & CaSR \\
- Gracile bone dysplasia (GCLEB) & GNA11 \\
Parathyroid-specific functional and developmental abnormalities & PTH, GCMB, SOX3 \\
type 5 & Autosomal dominant hypocalcemia with hypercalciuria type 1 (ADH1)/Bartter syndrome \\
- Autosomal dominant hypocalcemia with hypercalciuria type 2 (ADH2) & - \\
- Familial isolated hypoparathyroidism (autosomal, X-linked) (FIH) & - \\
Immune system abnormalities & \\
- Autoimmune polyendocrine syndrome type 1 (APS1) & Mitochondrial DNA \\
- Autoimmune polyendocrine syndrome type 4 (APS4) & Mitochondrial DNA \\
Mitochondrial diseases & \\
- Kearns-Sayre syndrome (KSS) & HADHA, HADHB \\
- Mitochondrial encephalomyopathy with lactic acidosis and stroke-like episodes & ACADM \\
- Mitochondrial trifunctional protein deficiency syndrome & DHCR7 \\
- Medium chain acylCOA dehydrogenase deficiency) &
\end{tabular}

mutations occurring later in development will be more specific for the parathyroid glands, causing isolated hypoparathyroidism.

Penetrance and expressivity are variable, even within subjects with a same mutation belonging to the same kindred. This high variability may be derives from interactions with environmental and genetic factors, which remain to be identified. Once established, the disease lasts life-long. Thus, even in the presence of mild hypocalcemia, ectopic calcifications are likely to occur. Dental abnormalities and bone dysplasia can be observed in the case of developmental disorders.

\section{Genetic isolated hypoparathyroidism}

Genetic isolated hypoparathyroidism encompasses a group of disorders, in which hypocalcemia ensuing from parathyroid insufficiency is the only biochemical and functional abnormality caused by a de novo or inherited mutation transmitted following an autosomal dominant, recessive or X-linked pattern of inheritance. Nonetheless, the majority of the cases of genetic hypoparathyroidism are sporadic. Isolated hypoparathyroidism may present with a wide range of disease severity, going from life-threatening, early onset, neonatal hypocalcemia to mostly asymptomatic disease $[4,9]$. They are usually manifest at birth or in the first decade of life as long as abnormalities in the PTH genes or in genes encoding for parathyroid-specific transcription factors are responsible for the disease (overall indicated with OMIM phenotype number \#146200). In this case the disorder is very rare (less than 1 case in 1,000,000 individuals). When a functional abnormality of the parathyroids due to an altered CaSR signaling is the cause, the disease has a higher prevalence (1:70,000 cases), usually milder clinical manifestations, so that it might manifest in adult life or remain undiagnosed [22].

Mutations in the transcription factor GCMB (encoded by GCM2, OMIM gene number *603716, chromosomal locus 6p24.2), one of the human hortologs of the Drosophila glial cell missing $(\mathrm{gcm})$, can 
be responsible of both of autosomal recessive or autosomal dominant familial isolated hypoparathyroidism. GCMB is a transcription factor mainly expressed in the developing and mature parathyroids and recognized as critical for parathyroid development, differentiation and function, as demonstrated in mice lacking Gcm2 [23]. It has been recently demonstrated also to regulate CaSR and PTH expression in mature parathyroid cells [24]. A homozygous intragenic deletion in GMC2 was first demonstrated in the proband belonging to large kindred with familial isolated hypoparathyroidism [25]. Another loss of function homozygous mutation in GCM2 was later shown in two siblings from asymptomatic, first cousin parents, in whom a residual PTH secretion could be demonstrated [26]. Further studies in families with autosomal recessive isolated hypoparathyroidism has characterised additional GCM2 mutations and shown that they can impair nuclear localization, DNA-binding or transactivational ability of this $\mathrm{j}$ key parathyroid transcription factor [27]. Autosomal dominant hypoparathyroidism can be caused by heterozygous, dominant-negative mutations in GMC2, which lead to the production of an aberrant GCMB protein usually impairing the transactivation capacity of the wild-type protein $[28,29]$. A study in Indian families affected from autosomal recessive hypoparathyroidism has demonstrated GCM2 mutations in up to $80 \%$ of the cases, even in the absence of consanguineity, maybe because of a founder effect [27].

Mutations in the preproPTH gene (OMIM gene number * 168450 , chromosomal locus 11p15.2) have described to be associated both to autosomal dominant and autosomal recessive hypoparathyroidism. A missense mutation in the sequence of the pre-proPTH gene encoding for the hydrophobic core of the signal peptide was first described as responsible of autosomal dominant familial isolated hypoparathyroidism [30]. This mutant protein is not properly processed through the endoplasmic reticulum. Thus, it accumulates within the cytoplasm, becomes toxic and promotes apoptosis of the parathyroid cell [31]. A homozygous mutation in the preproPTH gene described in kindreds with autosomal recessive hypoparathyroidism has been shown to lead to aberrant preproPTH mRNA devoid of exon 2, which contains the sequence for the signal peptide and initiation codon for protein translation $[32,33]$. Other homozygous mutations in exon 2 of the preproPTH gene similarly lead to aberrant or truncated PTH propeptides impairing intracellular PTH trafficking and maturation within the parathyroid cell and are transmitted as an autosomal recessive trait, indicating exon 2 as a probable hot spot for mutations in the preproPTH gene [34].

Some forms of early-onset, severe hypoparathyroidism due to parathyroid agenesis can be transmitted as a X-linked recessive disease, thus occurring in only in males. It is a very rare condition and it has been described just in two kindreds from the United States [35]. Chromosomal rearrangements involving chromosomes 2p25 and Xq27 likely affect the expression of SOX3, a SRY (sex-determining gene on chromosome $\mathrm{Y}$ ) related high-mobility group has been demonstrated. This gene is expressed early in developing parathyroids and is likely to regulate their growth and differentiation [36].

Recently, a compound heterozygous mutation in the autoimmune regulator gene (AIRE, OMIM gene number ${ }^{*} 607358$, chromosomal locus 21q22.3), a gene responsible for autoimmune endocrinopathy type 1 (APS1, see paragraph 2.1.2) has recently been demonstrated to be also the cause of autosomal recessive isolated hypoparathyroidism [37].

Mild variants of isolated hypoparathyroidism are likely to be caused by partial inactivation of CaSR signaling [22]. Gain-of-function mutations of CaSR (OMIM number *601199 on chromosome 3q21) cause the disorder named as autosomal dominant hypocalcemia with hypercalciuria (ADH1 or HYPOC1, OMIM phenotype number \#601198), also referred to as familial hypercalciuric hypocalcemia $[38,39]$. De novo or familial heterozygous mutations in the CaSR gene may lead to a partially constitutive active CaSR, which inhibits PTH transcription and secretion (PTH levels are usually in the lownormal range), thus inducing congenital mild hypocalcemia. At the same time, fractional calcium excretion is increased, leading to with a tendency towards or frankly overt hypercalciuria [40]. This is the reason why the individuals with this disorder are likely to develop nephrolithiasis, renal and soft tissue ectopic calcifications. Hypocalcemic symptoms are generally mild or absent, with few episodes related to muscle hyperexcitability. Serum levels of magnesium are typically in the low-normal range and may worsen the clinical manifestations. The phenotype of a Bartter-like syndrome with hypokalemic alkalosis, hyperreninemic hyperaldosteronism (Bartter syndrome type 5) can accompany the mineral disturbances in the case of specific gain-of-function mutations of CaSR [22]. 
Gain-of-function mutations of the $\alpha$-subunit of the guanine nucleotide-binding protein G11 (G $\alpha q / 11$, OMIM gene number ${ }^{*} 139313$ on chromosome 19p13) cause ADH2 or HYPOC2 (OMIM phenotype number \#615361) [41,42]. Gaq/11 is a membrane-associated protein mediating CaSR signal transduction intracellularly. The phenotype of $\mathrm{ADH} 2$ resembles that of $\mathrm{ADH} 1$, with mild, often asymptomatic hypocalcemia, but it is usually associated with a milder degree of hypercalciuria and a overall milder renal phenotype. Sometimes, individuals with ADH2 can present a skeletal phenotype characterized by short stature, pointing to a possible role of Gaq/11 in development and/or postnatal growth [43].

Since the majority of patients with ADH are asymptomatic or display mild symptoms, they do not typically require conventional treatment with calcium salt and active vitamin D metabolites, which is indeed indicated in the case of symptomatic hypocalcemia [22]. Nonetheless, the dosage regimens has to be carefully monitored and maintained to the minimum dose effective in controlling symptoms for the likelihood of worsening hypercalciuria with polyuria and polydipsia, inducing nephrolithiasis, and nephrocalcinosis/renal failure in the long-term. Thiazide diuretics may be employed in order to decrease calciuria. CaSR antagonists (i.e. calcilytics), allosteric modulators of the CaSR, have been indicated as the therapy of choice of $\mathrm{ADH}$, for their effect of increasing serum calcium and at the same time limiting urinary calcium excretion [44].

\section{Genetic syndromic hypoparathyroidism}

Hypoparathyroidism may occur within complex syndromes and thus appear in association with other clinical manifestations not related to parathyroid-driven mineral disturbances. The majority of these syndromes derive from developmental abnormalities, which may also involve organs arising from the third and fourth pharyngeal pouches [9]. In some cases hypoparathyroidism can be associated to skeletal dysplasia or cranial malformations, other malformations, or autoimmune diseases such within monogenic autoimmune disorders such as APS1. Rarely, hypoparathyroidism can represent one of the features belonging to the complex clinical picture belonging to mitochondrial diseases [9].

DiGeorge syndrome type 1 and 2 (DGS1 and 2, OMIM phenotype number \#188400 and \#601362, respectively) are complex conditions determined by chromosomal abnormalities, i.e. a hemizygous microdeletions in chromosome 22q11.2 or 10p13-14 (OMIM locus number ${ }^{*} 602054$ and ${ }^{*} 601362$, respectively), ensuing de novo or inherited as an autosomal dominant trait [45,46]. Recently, in a severe case of DGS with progressive renal failure, a double deletion in 22q11.2 and 10p13-14 has been detected. Each of these chromosomal abnormalities determines developmental abnormalities in the formation in the derivatives of the third and fourth pharyngeal pouches. The deleted region in DGS1 contains TBX1, whose transcript, T-box 1, is crucial for the development of thymus and the parathyroids and its haploinsufficiency could be responsible for the DGS phenotype [47]. Indeed, in some cases of DGS negative for chromosomal deletions, loss-of-function mutations in TBX1 have been reported. TBX1 transcript appears to enhance GCMB expression, as demonstrated in mice TBX1 knockout in which GCMB is downregulated. The chromosomal locus for DGS2 contains NEBL, encoding nebulette, whose role in parathyroid development has to be clarified. A great phenotypic variability is characteristic of DGS, which has a prevalence of 1:2000-4000 in the general population and represents the most common microdeletion disease in humans [45]. Since specific circulating microRNAs signature has been described in DGS, it has been hypothesized that circulating microRNAs pattern could be responsible for DGS phenotype variability [48]. Chromosomal deletions in 22q11.2 are responsible for $90 \%$ of cases of DGS (i.e. DGS1). DGS1 is also referred to as "CATCH 22", being characterized by cardiac and craniofacial abnormalities, T-cell deficit due to thymic hypoplasia, cleft palate, hypocalcemia due to hypoparathyroidism) [45]. Immunological abnormalities including both T-lymphocyte and B-lymphocytes deficiency, anatomic abnormalities of the heart and great vessels, and typical facies with mouth, nose and eye defects, along with usually mild cognitive impairment and increased risk of neurologic diseases such as autism and schizophrenia can be part of the clinical picture [49]. DGS is the most common cause of palatal abnormalities and the second most common cause of congenital heart diseases and developmental delay. A variable degree of penetrance and severity characterizes DGSrelated hypoparathyroidism, according to the degree of parathyroid diagnosis, without any genotype-to-phenotype correlation. On one side of the clinical spectrum there is severe hypocalcemia due to parathyroid agenesis, which may be present at birth. At the other side of the spectrum there is mild hypocalcemia due to parathyroid hypoplasia, which can manifest in adulthood [45,50]. The 
chromosomal abnormalities causing DGS1 and DGS2 can be simply detected by cytogenetic analysis [51]. Given the relative high frequency and the simple cytogenetic screening, DGS has to be always excluded in the screening for idiopathic hypoparathyroidism, even in apparently isolated forms. In some cases, patients diagnosed with DGS can display features belonging to CHARGE syndrome (OMIM phenotype number \#214800) [52]. In this disorder, eye and hear abnormalities, genitourinary and heart disturbances, growth retardation can constitute typical additional features. It is due to heterozygous mutations in chromodomain helicase DNA-binding 7 (CHD7, OMIM gene number *608892) or in semaphorin 3-E gene (SEMA3E, OMIM gene number *608166), whose pharyngeal ectoderm specific transcripts have key function in the development of pharyngeal-derived organs [53].

Syndromic hypoparathyroidism can be generated by alterations of various transcription factors encoding proteins, whose activity is located upstream GCMB, PTH or CaSR [9].

The gene GATA3 (OMIM gene number *131320, chromosomal locus 10p14) is a zinc finger transcription factor expressed early in developing kidneys, inner ear, central nervous system, thymus, hematopoietic cell lineages and parathyroids [8]. In the parathyroid cells it regulates the expression of $G C M B$ and PTH [54]. Heterozygous loss-of-function mutations or dominant-negative in this gene, which can be inherited in an autosomal dominant fashion, generate the hypoparathyroidism, deafness and renal dysplasia syndrome (HDR, OMIM phenotype number \#146255), also referred to as Barakat syndrome or nephrosis, nerve deafness and hypoparathyroidism syndrome [55]. This syndrome is characterized by the occurrence of mild-to-severe hypoparathyroidism (according to the degree of parathyroid aplasia-hypoplasia), together with moderate-to-severe dysplastic renal alterations, bilateral symmetrical sensorineural deafness. Other features such as neurological symptoms, anatomic abnormalities of the genital tract and intestine, craniofacial malformations can be associated to the classical triad. More than 90\% of GATA3 mutations are clustered within the sequence encoding the Nterminal zinc finger (ZnF1), whose function is to increase DNA binding affinity [56]. No genotype-tophenotype correlation exists, since the same GATA3 mutation can originate various clinical combinations of hypoparathyroidism, deafness and renal dysplasia, with different grades of severity, in particular as far as renal abnormalities are concerned [57]. Indeed, while hypoparathyroidism and deafness have a penetrance greater than $90 \%$, renal dysplasia has a penetrance less than $80 \%$. In any case, so far, GATA3 mutations seem to be responsible of cases of isolated hypoparathyroidism.

Syndromic hypoparathyroidism may occur in association with signs of skeletal dysplasia or dysmorphism.

Both hypoparathyroidism, retardation and dysmorphism syndrome (HRDS, OMIM phenotype number \#241410, also referred to as Sanjad-Sakati syndrome) and Kenny-Caffey syndrome type 1 (KCS1, OMIM phenotype number \#244460) are due to homozygous mutations in tubulin-specific chaperone E (TBCE, OMIM gene number *604934), a molecular chaperone potentially implicated in parathyroid gland migration and bone development [58]. In both the disorders, inherited as autosomal recessive trait, affected individuals display a proportionate short stature (dwarfism) associated with hypoparathyroidism and ocular abnormalities (papilledema, retinal vascular tortuosity, corneal opacity, recurrent infections). In KCS1 bones appear overall small and thin, although cortices of the long bones are thick at X-ray imaging with medullary stenosis. Vertebrae and pelvic bones are typically spared. Macrocephaly due to osteosclerosis of the skull, with forehead prominence, and defective dentition (short roots, hypo-oligodontia or microdontia) are associated features. In HRDS, described in kindreds from the Middle East, signs of osteosclerosis are usually missing, while a typical facial dysmorphism (deep-set eyes, abnormal external ears, frontal bossing) is present. In HRDS pituitary hormone deficiencies may be associated [59].

A very sever phenotype similar to KCS1, with high perinatal lethality, is gracile bone dysplasia (GCLEB, OMIM phenotype number \#602361) [60]. These newborns display thin long bones, with stenosis of medullary cavity, and pronounced osteocraniostenosis (OCS), with triangular face with frontal bossing and microphtalmia. Signs of hypoparathyroidism may appear in few infants surviving the newborn period. This disorder is caused by a heterozygous deletion (Ser342del) in the gene encoding family with sequence similarity 111 member A (FAM111A, OMIM gene number *605292), whose product has a role in DNA replication and chromatin maturation [60]. Heterozygous missense mutations in FAM111A (Arg569His) have been described as the cause of Kenny-Caffey syndrome type 2 
(KSC2, OMIM phenotype number \#127000), a disease sharing a similar phenotype with KCS1 but inherited in an autosomal dominant fashion [61].

Hypoparathyroidism, as well as other endocrinopathies (i.e. hypogonadism, growth hormone deficiency, diabetes mellitus) can be sometimes a feature of the complex phenotype of mitochondrial diseases such as mitochondrial myopathy, encephalopathy, lactic acidosis, and stroke-like episodes (MELAS, OMIM phenotype number \#540000) and Kearns-Sayre syndrome (KSS, OMIM phenotype number \#530000), due to mutations, deletions or duplications in mitochondrial DNA (mtDNA) [62]. Other mitochondrial disorders potentially featuring hypoparathyroidism are the medium chain acylCoA dehydrogenase deficiency (ACADMD, OMIM phenotype number \#201450), caused by mutations of medium chain acylCoA dehydrogenase gene (ACADM, OMIM gene number *607008 on chromosome 1p31.1) and mitochondrial trifunctional protein deficiency (MTPD, OMIM phenotype number \#609015), caused by homozygous or compound heterozygous mutations in the genes encoding the $\alpha$ subunit or the $\beta$-subunit of the mitochondrial trifunctional protein (HADHA and HADHB, OMIM gene numbers *600890 and *143450, respectively, on chromosome 2p23.3) [62]. This protein is responsible for the $\beta$-oxidation of fatty acids [63].

In all these mitochondrial diseases, the early-onset clinical picture is mainly related to the specific, usually severe manifestations, although hypoparathyroidism and the other possible endocrinopaties have to be ruled out in individuals with who survive into childhood and later stages [62].

Recently, hypoparathyroidism due to parathyroid hypoplasia has been described in some individuals with Smith-Lemli-Opitz syndrome (SLOS, OMIM phenotype number \#270400), a metabolic disorder characterized by reduced cholesterol biosynthesis and typical congenital malformations (microcephaly with short nose, altered development of male genitalia, renal dysplasia, syndactyly), and mental retardation. This disorder is caused by homozygous mutations in the DHCR7 gene (OMIM gene number ${ }^{*} 602858$ on chromosome 11q13.4) leading to reduced cholesterol biosynthesis [64].

Genetically-determined, syndromic hypoparathyroidism can be part of a polyendocrine syndrome, namely autoimmune polyendocrine syndrome type 1 (APS1, OMIM phenotype number \#240300), also named as autoimmune polyendocrinopathy-candidiasis-ectodermal dystrophy (APECED). APS1 is a monogenic disorder caused by inactivating mutations of the autoimmune regulator gene (AIRE, OMIM gene number *607358 on chromosome 21q22.3) [65]. APS1 prevalence can be higher in genetically isolated populations such as Finnish, Iranian Jewish, and Sardinians because of a founder effect. AIRE is expressed early in the thymus and has a key role in promoting initial immunological tolerance to selfantigens [66]. APS1 is inherited as an autosomal recessive trait, although even dominant-negative mutations, inherited following an autosomal dominant pattern of inheritance, have been described as responsible of the syndrome. The triad of candidiasis, hypoparathyroidism, and Addison's disease, usually occurring in this order, characterizes APS1 [65]. Mucocutaneal candidiasis caused by T-cell immunodeficiency, hypoparathyroidism and adrenal insufficiency usually occur at early stages, first and second-third decades of life, respectively. Additional autoimmune endocrine and non-endocrine autoimmune disorders (i.e. insulin dependent diabetes mellitus, autoimmune thyroid diseases, gonadal failure, pernicious anemia, celiac disease, vitiligo, alopecia) can be associated manifestations. Ectodermal dystrophies represented by dental abnormalities may occur [65]. Chronic inflammatory autoreactive T-cell infiltrate can be demonstrated in affected organs as well as organ-specific autoantibodies. Indeed, the positivity of 21-hydroxylase antibodies strongly indicates a high probability of developing adrenal insufficiency. Antibodies against T-helper type 17 cell-related cytokines and against type I interferons (IFN- $\alpha$ and IFN- $\omega$ ) have been indicated as specific feature and a marker of APS1, although not causative, likely directly correlated to the susceptibility to Candida infections and possible protection against autoimmune-induced organ damage [67]. Conversely, autoantibodies against the extracellular domain of the calcium sensing receptor (CaSRAbs) and against parathyroid autoantigens such as NACTH leucine-rich-repeat protein 5 (NALP5Abs) have been demonstrated only in some cases of APS1-related hypoparathyroidism, so that they cannot be considered a marker of the disease $[68,69]$. Overall, a wide spectrum of APS1 phenotypic variability exists, without any verifiable genotype-tophenotype correlation. Previously, the diagnosis of APS1 was made on the presence of two components of the triad and, within affected kindred, the presence of at least one major endocrine abnormality. Since hypoparathyroidism has a high penetrance (100\% in females and $60 \%$ in men), according to a recent consensus, the occurrence of hypoparathyroidism together with another manifestation of 
the triad, AIRE mutation or the presence of specific antibodies is necessary for the diagnosis of APS1 [70].

Non-genetic hypoparathyroidism

Non-genetic hypoparathyroidism include the most common forms of hypoparathyroidism in clinical practice, such as hypoparathyroidism occurring after neck surgery and non-genetic autoimmune hypoparathyroidism. In the case of non-genetic hypoparathyroidism, and in particular surgical hypoparathyroidism, clinical history can easily drive the diagnosis. Since no specific marker exists in nongenetic autoimmune hypoparathyroidism, the diagnosis of this disorder is a diagnosis of exclusion, or indirectly made through the concurrent presence of other autoimmune diseases.

\section{Non-genetically determined autoimmune hypoparathyroidism}

Non-genetic, autoimmune hypoparathyroidism may manifest as an isolated disorder or, more frequently, in combination with other autoimmune conditions as it occurs in autoimmune polyendocrinopathy syndromes (APSs) such as APS type 3, when associated with autoimmune thyroid diseases, or APS4, when occurring in combination with other non-thyroid autoimmune diseases [11]. Autoantibodies against parathyroid autoantigens (NALP5Abs and CaSRAbs) have been described as associated to autoimmune hypoparathyroidism, but their specificity and sensitivity for diagnosis of autoimmune hypoparathyroidism is low. In addition, it is not clear whether they can be simply considered an epiphenomenon or marker of the disease or whether they have a real pathogenetic role [11,71]. For this reason they are not assessed routinely in clinical practice. On the other hand, the assessment of other organ-specific autoantibodies in the presence of established idiopathic hypoparathyroidism should be advised to indirectly suggest a possible autoimmune pathogenesis of the disorder.

Although no definitive conclusion can be derived on the prevalence of the disease for the absence of specific markers, it is today estimated that autoimmune hypoparathyroidism is overall the most prevalent form of idiopathic hypoparathyroidism.

\section{Post surgical hypoparathyroidism}

Hypoparathyroidism ensuing as a complication of neck surgery and thyroid surgery, in particular, is the most usual form of hypoparathyroidism. The parathyroids lay in close proximity to the thyroid and share with it the same blood supply. The parathyroids can be devascularized, damaged thermically by the bovie, or unintentionally removed during thyroid surgery or large neck dissections for infiltrative diseases [10]. Hypoparathyroidism is the obvious consequence of subtotal parathyroidectomy for parathyroid hyperplasia, while it occurs rarely after partial removal of the parathyroids for the high functional reserve of these glands [72,73]. Hypoparathyroidism is defined as transient when lasting less than 6 months/usually few weeks) after surgery. Chronic or permanent hypoparathyroidism refers to an insufficient PTH secretion persisting for more than 6 months after the surgical procedure [10].

The incidence of hypoparathyroidism after surgery strongly depends upon the extent of the intervention and the experience of the neck surgeon. Usually, the probability of hypoparathyroidism increases after total, subtotal or near total thyroidectomy performed in the case of thyroid cancer, lymph node or radical neck dissections for other neck malignancies, or benign thyroid diseases such as large multinodular goiters and Graves' disease. Conversely, partial thyroidectomy is infrequently associated with hypoparathyroidism $[10,72,73]$.

The experience of an endocrine surgeon is another major determinant of the incidence of surgical hypoparathyroidism. An endocrine surgeon is considered "experienced" when he/she has performed more than 100 thyroidectomies [10,72,73]. In tertiary referral centers for endocrine surgery the rate of transient and permanent surgical hypoparathyroidism is typically low (5.4-9.6\% and $0.5-1.7 \%$, respectively) [74]. In epidemiological studies performed by retrieving data from large proprietary insurance databases, the estimated incidence of hypoparathyroidism after neck surgery was 1.9\% [14]. This rate was similar to the one obtained European Mediterranean countries such as Italy through the indirect estimate of cases of hypoparathyroidism as the ones on chronic treatment with active vitamin 
D [18]. In Denmark the overall prevalence of surgical hypoparathyroidism was 22 per 100,000 inhabitants [15]. In children undergoing neck surgery, the incidence of hypoparathyroidism after neck surgery can be as high as $7.3 \%$, even if the operation has been performed in endocrine surgery referral centers [75].

A number of studies have attempted to identify possible predictive factors of postoperative hypoparathyroidism [76]. Preoperative serum calcium and 25 hydroxyvitamin D levels, low intraoperative $\mathrm{PTH}$, have been documented as independent risk factors for transient postsurgical hypoparathyroidism. As far as postoperative indexes are concerned, the determination of serum calcium and/or PTH levels in the first day after surgery has been suggested as predictive of hypoparathyroidism $[77,78]$. However, hypocalcemia may also occur or worsen some days after surgery. Therefore, it is important to obtain a measurement of albumin-corrected serum calcium levels even on the 3rd-4th day after the surgical procedure. Regarding the surgical procedure itself, the failure to identify at least 2 parathyroid glands during neck surgery has been independently associated with the risk of developing subsequent permanent parathyroid insufficiency $[10,72,73]$.

\section{Diagnostic assessment in hypoparathyroidism}

Different diagnostic issues have to be considered in the various forms of hypoparathyroidism. The diagnosis of previously defined idiopathic forms is still a challenge, but the precise classification with the genetic cause guides the diagnostic workup. At the same time, the assessment for possible associated non parathyroid-related disturbances helps in refining the diagnosis and at the same time in better defining the clinical picture [9]. For instance, in cases such as ADH, the analysis of CaSR or GNA11 is of key importance to better plan the long-term treatment and avoid complications related to conventional therapy.

Surgical hypoparathyroidism does not usually pose big issues regarding the diagnosis, but it requires an accurate definition of the type (transient vs permanent), even in apparent established cases [Marcocci 2012].

Functional hypoparathyroidism (i.e. magnesium disturbances) has always to be excluded in all the possible forms of hypoparathyroidism. Indeed, besides representing a possible cause of hypoparathyroidism by itself, it can also worsen hypoparathyroid-related symptoms [12].

\section{Diagnostic framework in non-surgical hypoparathyroidism}

In Fig. 2, a diagnostic workup of non-surgical hypoparathyroidism is proposed. The clinical picture drives the genetic hypothesis. The complete clinical assessment looking for possible association of organ involvement other than the parathyroids is fundamental for the diagnosis of isolated or syndromic hypoparathyroidism. The measurement of corrected serum calcium or ionized calcium levels in first-degree relatives of a patient with idiopathic hypoparathyroidism is necessary to exclude or confirm familial forms [9]. Despite the increasing knowledge in this area, the diagnosis of these forms remains difficult particularly when patients are not referred to centers of expertise, where specific genetic diagnostic workup protocols are carried out.

Given the high frequency of DiGeorge syndrome in the general population, cytogenetic analysis to screen for possible microdeletions on chromosomes 22 or 10 are always recommended and represent an easy test to perform in clinical practice, even in the case of adults with apparently isolated hypoparathyroidism, in whom overt anatomical abnormalities or immunodeficiencies are not detected [Palmer 2018]. In cases of childhood-onset hypoparathyroidism sequence of the major genes involved in the pathogenesis of hypoparathyroidism can unveil a mutation in one of the genes CaSR/GNA11, $G C M B$, and GATA3 in up to $35 \%$ of patients. Sequence analysis of AIRE can be useful also in cases of isolated hypoparathyroidism or, even more so, when the APS1-related triad is recognized. In severe, early-onset cases, the sequence of $P T H$ gene can be undertaken. If signs of skeletal dysplasia are recognizable, the analysis of TBCE, FAM111A, is advisable.

Genetic testing in non-surgical hypoparathyroidism is important to confirm the clinical diagnosis, to implement the appropriate treatment, to identify family members carriers for a specific mutation. 


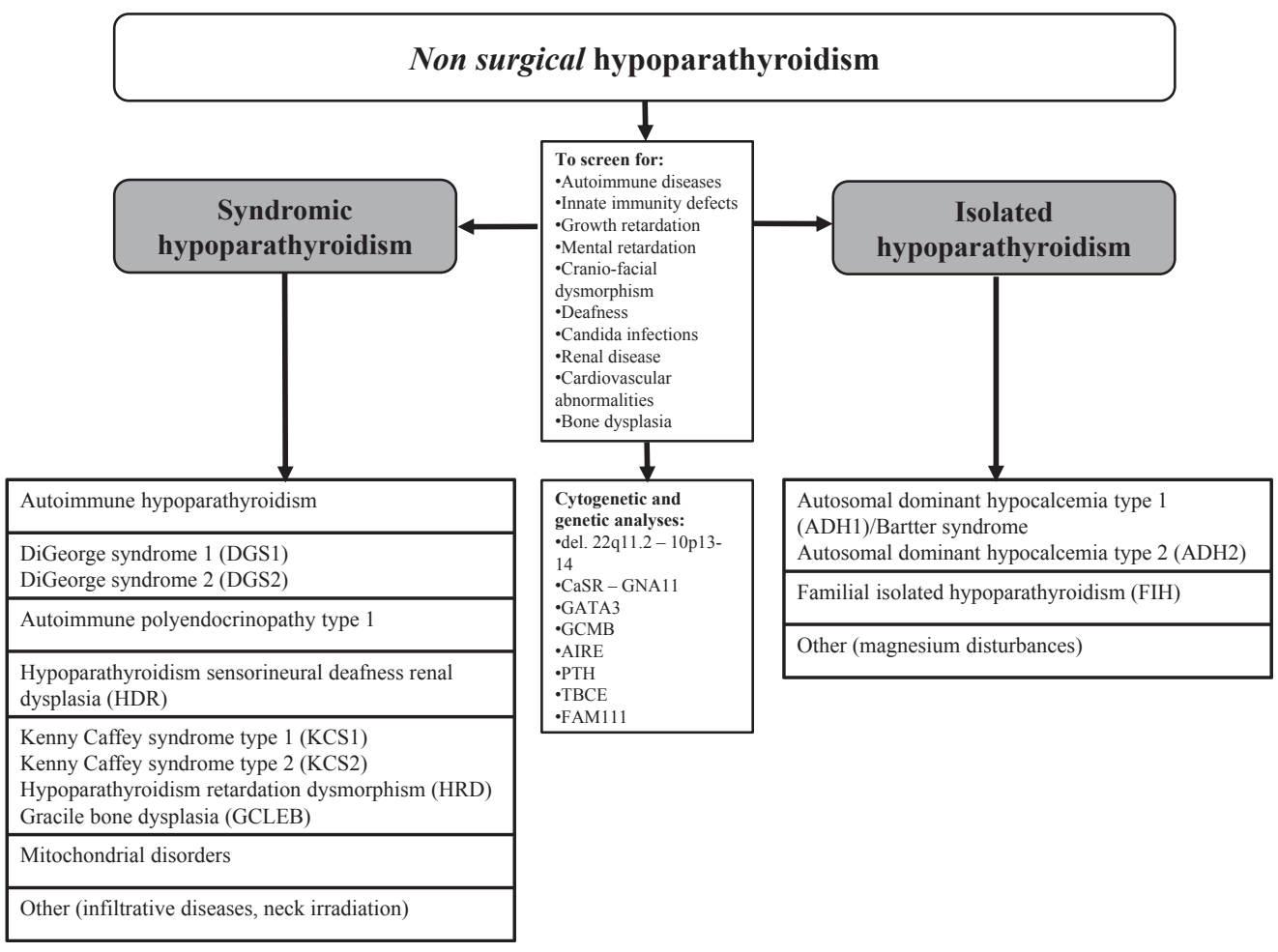

Fig. 2. Proposed flow chart for the diagnosis of non-surgical forms of hypoparathyroidism. The majority of non-surgical forms refer to genetic hypoparathyroidism (see text for further specifications).

\section{Diagnostic framework in surgical hypoparathyroidism}

The diagnosis of surgical hypoparathyroidism poses the important issues for the proper detection of hypoparathyroidism itself and of the possible transient forms, which do not require life-long therapy with calcium and active vitamin D metabolites.

In Fig. 3 a diagnostic workup for postsurgical hypocalcemia, and, in particular, for postsurgical hypoparathyroidism, is proposed. Patients undergoing neck surgery have always to be checked for serum calcium and the possible presence of hypocalcemic signs or symptoms have to be ruled out. In the case of hypocalcemia occurring within few days after neck surgery, low serum phosphate levels and/or high PTH indicate a PTH-independent cause of hypocalcemia. In this case, a postoperative hungry bone syndrome, due to the rapid normalization of a high bone turnover state reverted to normal by surgical therapy with consequent fast bone mineral accrual (i.e. hyperthyroidism, hyperparathyroidism), is likely. This state spontaneously resolves within few months after surgery and it is temporary by definition. High serum phosphate and inappropriately low or undetectable PTH levels indicate surgical hypoparathyroidism, with the need to start appropriate treatment with calcium salts and active vitamin D [10]. Even in patients under long-term replacement therapy, transient forms of hypoparathyroidism have to be ruled out, especially in cases in which PTH is in the low-normal range, calcemia is in the upper normal range with normal phosphatemia. Indeed, low or untraceable PTH levels under chronic treatment with $1 \alpha$-hydroxylated vitamin D metabolites do not exclude transient hypoparathyroidism, since these drugs may suppress the possible residual PTH secretion. In the case normocalcemia is maintained under down titration till the discontinuation of the replacement therapy, permanent/chronic hypoparathyroidism can be excluded in order to avoid unnecessary long-term therapy $[7,10]$. 


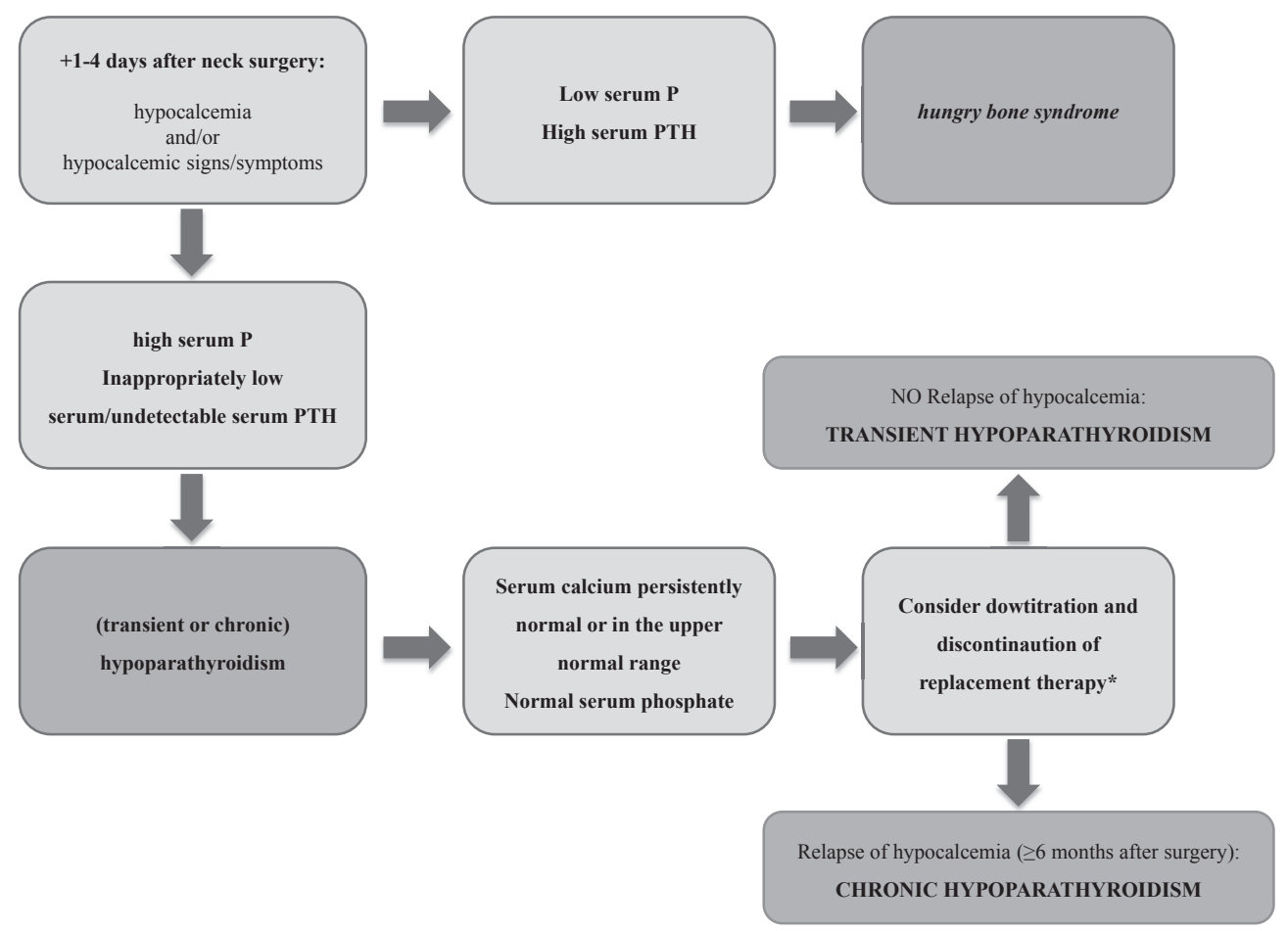

Fig. 3. Proposed diagnostic framework in post-surgical hypoparathyroidism (see text for further specifications); * calcium salts and active vitamin D metabolites.

\section{Conclusions}

Pathophysiology of hypoparathyroidism is complex and requires a multifaceted approach. Both surgical and non-surgical forms pose diagnostic challenges. A clinical assessment to exclude syndromic forms even in cases of apparent isolated disease, is always necessary in non-surgical, idiopathic hypoparathyroidism. The specific diagnostic testing is strongly driven by a proper clinical assessment to exclude/confirm syndromic forms, even in cases of apparent isolated hypoparathyroidism. Transient post-surgical hypoparathyroidism have to be ruled out, even in patients on long-term therapy with calcium salts and active vitamin D. Since no highly specific and sensitive marker of parathyroid autoimmunity exists, the diagnosis of autoimmune hypoparathyroidism is still a diagnosis of exclusion.

The proper definition of the etiopathogenesis of hypoparathyroidism has contributed a lot to better understanding of parathyroid physiology and is fundamental to any therapeutic approach.

\section{Summary}

The terms hypoparathyroidism refers to a broad group of diseases of different etiology, all characterized by the deficient levels of PTH leading to hypocalcemia and hyperphosphatemia, requiring chronic replacement of calcium and active vitamin D. Besides post-surgical hypoparathyroidism, which poses a diagnostic challenge as far as the recognition of transient forms are concerned, the development of molecular endocrinology during the last two decades has greatly improved the diagnosis of forms once names as idiopathic. The identification of genes responsible of syndromic and isolated forms of non-surgical hypoparathyroidism has also improved the understanding of parathyroid physiology. Epidemiological data on the prevalence, incidence and natural history of the disease are still scarce and further cross-sectional and longitudinal studies are needed. 
Protocols of systematic clinical and genetic assessment in idiopathic forms are still lacking. They are indeed necessary, in order to plan the correct therapy and decrease the complications in the long term (i.e. soft tissue calcifications, nephrolithiasis, nephrocalcinosis). Clinical assessment in non-surgical forms is particularly important, since it can unveil associated, mainly asymptomatic non parathyroid-related abnormalities pointing to syndromic hypoparathyroidism. Autoimmune hypoparathyroidism, which represents a substantial portion of the non-surgical forms, still does not have a proper marker for diagnosis and, for this reason, is usually a diagnosis of exclusion.

\section{Practice points}

- Hypoparathyroidism is complex disorder of mineral metabolism, whose physiopathology, driven by the absence of proper PTH actions on a number of tissues and organs, include many disturbances beyond the abnormal calcium homeostasis.

- With a mean prevalence of $25-30$ cases in 100,000 inhabitants, hypoparathyroidism is overall considered a rare disease, although the incidence of post-surgical hypoparathyroidism can be higher in some settings, depending on the experience of the surgeon.

- Clinical screening of syndromic hypoparathyroidism is always recommended, even in cases of apparently isolated hypoparathyroidism, both in children and in adults.

- Cytogenetic and genetic analyses have always to be undertaken in order to better diagnose cases of idiopathic hypoparathyroidism and offer proper genetic counseling.

- It is mandatory to assess calcemia (and phosphatemia) within 1-4 days after neck surgery, even in asymptomatic patients.

- Transient postsurgical hypoparathyroidism has to be ruled out even in supposedly permanent/established hypoparathyroidism, in order to discontinue unnecessary long-term treatment with calcium salts and active vitamin $D$, which can lead to long-term complications (soft tissue calcifications, nephrolithiasis, nephrocalcinosis).

\section{Research agenda}

- Epidemiological data on the prevalence and incidence as well as on natural history of hypoparathyroidism are still needed in a number of settings.

- Shared protocols for structured clinical assessment in non-surgical forms are needed.

- Automated screening techniques of common genes in idiopathic hypoparathyroidism have to be developed for differential diagnosis of non-surgical hypoparathyroidism.

- Better characterization of autoimmune hypoparathyroidism, with the finding of a specific and sensitive marker has to be pursued.

\section{References}

[1] Shoback D. Clinical practice. Hypoparathyroidism. N Engl J Med 2008;359:391-403.

*[2] Brandi ML, Bilezikian JP, Shoback D, et al. Management of hypoparathyroidism: summary statement and guidelines. J Clin Endocrinol Metab 2016;101:2273-83.

*[3] Bollerslev J, Rejnmark L, Marcocci C, et al., European Society of Endocrinology. European society of endocrinology clinical guideline: treatment of chronic hypoparathyroidism in adults. Eur J Endocrinol 2015;173:G1-20.

*[4] Clarke BL, Brown EM, Collins MT, et al. Epidemiology and diagnosis of hypoparathyroidism. J Clin Endocrinol Metab 2016; 101:2284-99.

[5] Cheloha RW, Gellman SH, Vilardaga JP, et al. PTH receptor-1 signalling-mechanistic insights and therapeutic prospects. Nat Rev Endocrinol 2015;11:712-24.

[6] Brown EM. Role of the calcium-sensing receptor in extracellular calcium homeostasis. Best Pract Res Clin Endocrinol Metab 2013;27:333-43. 
[7] Shoback DM, Bilezikian JP, Costa AG, et al. Presentation of hypoparathyroidism: etiologies and clinical features. J Clin Endocrinol Metab 2016;101:2300-12.

*[8] Grigorieva IV, Thakker RV. Transcription factors in parathyroid development: lessons from hypoparathyroid disorders. Ann N Y Acad Sci 2011;1237:24-38.

*[9] Cianferotti L, Brandi ML. The molecular genetics of hypoparathyroidism. In: Bilezikian JP, Marcus R, Levine MA, et al., editors. The Parathyroids: basic and clinical concepts. 3rd edn, Elsevier. London, Waltham San Diego: Academic Press; 2015. p. 719-36.

*[10] Marcocci C, Cianferotti L. The parathyroids. In: Miccoli P, Terris DJ, Minuto MN, et al., editors. Thyroid surgery: preventing and managing complications. Elsevier. Chichester: John Wiley \& Sons, Ltd; 2013. p. 227-36.

[11] Betterle C, Garelli S, Presotto F. Diagnosis and classification of autoimmune parathyroid disease. Autoimmun Rev 2014;13: $417-22$.

[12] Klein GL. Magnesium depletion and parathyroid function. In: Bilezikian JP, Marcus R, Levine MA, et al., editors. The Parathyroids: basic and clinical concepts. 3rd edn, Elsevier. London, Waltham San Diego: Academic Press; 2015. p. 697-706.

[13] Mannstadt M, Bilezikian JP, Thakker RV, et al. Hypoparathyroidism. Nat Rev Dis Prim 2017;3:17080.

[14] Powers J, Joy K, Ruscio A, et al. Prevalence and incidence of hypoparathyroidism in the United States using a large claims database. J Bone Miner Res 2013;28:2570-6.

[15] Underbjerg L, Sikjaer T, Mosekilde L, et al. Postsurgical hypoparathyroidism-risk of fractures, psychiatric diseases, cancer, cataract, and infections. J Bone Miner Res 2014;29:2504-10.

[16] Underbjerg L, Sikjaer T, Mosekilde L, et al. The epidemiology of nonsurgical hypoparathyroidism in Denmark: a nationwide case finding study. J Bone Miner Res 2015;30:1738-44.

[17] Cipriani C, Pepe J, Biamonte F, et al. The epidemiology of hypoparathyroidism in Italy: an 8-year register-based study. Calcif Tissue Int 2017;100:278-85.

[18] Cianferotti L, Parri S, Gronchi G, et al. Prevalence of chronic hypoparathyroidism in a mediterranean region as estimated by the analysis of anonymous healthcare database. Calcif Tissue Int 2018. https://doi.org/10.1007/s00223-0180405-5.

*[19] Kim JH, Shin YL, Yang S, et al. Diverse genetic aetiologies and clinical outcomes of paediatric hypoparathyroidism. Clin Endocrinol (Oxford) 2015;83:790-6.

[20] Clarke BL, Leibson C, Emerson J, et al. Co-morbid-medical conditions associated with prevalent hypoparathyroidism: a population-based study. J Bone Miner Res 2011;26:S182.

[21] Astor MC, Løvås K, Debowska A, et al. Epidemiology and health-related quality of life in hypoparathyroidism in Norway. J Clin Endocrinol Metab 2016;101:3045-53.

*[22] Hannan FM, Babinsky VN, Thakker RV. Disorders of the calcium-sensing receptor and partner proteins: insights into the molecular basis of calcium homeostasis. J Mol Endocrinol 2016;57. 1R127-42.

[23] Yuan Z, Opas EE, Vrikshajanani C, et al. Generation of mice encoding a conditional null allele of Gcm2. Transgenic Res 2014;23:631-41.

[24] Hendy GN, Canaff L. Calcium-sensing receptor gene: regulation of expression. Front Physiol 2016;7:394.

[25] Ding C, Buckingham B, Levine MA. Familial isolated hypoparathyroidism caused by a mutation in the gene for the transcription factor GCMB. J Clin Invest 2001;108:1215-20.

[26] Thomée C, Schubert SW, Parma J, et al. GCMB mutation in familial isolated hypoparathyroidism with residual secretion of parathyroid hormone. J Clin Endocrinol Metab 2005;90:2487-92.

[27] Bowl MR, Mirczuk SM, Grigorieva IV, et al. Identification and characterization of novel parathyroid-specific transcription factor Glial Cells Missing Homolog B (GCMB) mutations in eight families with autosomal recessive hypoparathyroidism. Hum Mol Genet 2010;19:2028-38.

[28] Mannstadt M, Bertrand G, Muresan M, et al. Dominant-negative GCMB mutations cause an autosomal dominant form of hypoparathyroidism. J Clin Endocrinol Metab 2008;93:3568-76.

[29] Mirczuk SM, Bowl MR, Nesbit MA, et al. A missense glial cells missing homolog B (GCMB) mutation, Asn502His, causes autosomal dominant hypoparathyroidism. J Clin Endocrinol Metab 2010;95:3512-6.

[30] Arnold A, Horst SA, Gardella TJ, et al. Mutation of the signal peptide-encoding region of the preproparathyroid hormone gene in familial isolated hypoparathyroidism. J Clin Invest 1990;86:1084-7.

[31] Karaplis AC, Lim SK, Baba H, et al. Inefficient membrane targeting, translocation, and proteolytic processing by signal peptidase of a mutant preproparathyroid hormone protein. J Biol Chem 1995;270:1629-35.

[32] Parkinson DB, Thakker RV. A donor splice site mutation in the parathyroid hormone gene is associated with autosomal recessive hypoparathyroidism. Nat Genet 1992;1:149-52.

[33] Sunthornthepvarakul T, Churesigaew S, Ngowngarmratana S. A novel mutation of the signal peptide of the preproparathyroid hormone gene associated with autosomal recessive familial isolated hypoparathyroidism. J Clin Endocrinol Metab 1999;84:3792-6.

[34] Ertl DA, Stary S, Streubel B, et al. A novel homozygous mutation in the parathyroid hormone gene (PTH) in a girl with isolated hypoparathyroidism. Bone 2012;51:629-32.

[35] Thakker RV, Davies KE, Whyte MP, et al. Mapping the gene causing X-linked recessive idiopathic hypoparathyroidism to Xq26-Xq27 by linkage studies. J Clin Invest 1990;86:40-5.

[36] Bowl MR, Nesbit MA, Harding B, et al. An interstitial deletion-insertion involving chromosomes 2p25.3 and Xq27.1, near SOX3, causes X-linked recessive hypoparathyroidism. J Clin Invest 2005;115:2822-31.

[37] Li D, Streeten EA, Chan A, et al. Exome sequencing reveals mutations in AIRE as a cause of isolated hypoparathyroidism. J Clin Endocrinol Metab 2017;102:1726-33.

[38] Pearce SH, Williamson C, Kifor O, et al. A familial syndrome of hypocalcemia with hypercalciuria due to mutations in the calcium-sensing receptor. N Engl J Med 1996;335:1115-22.

[39] Baron J, Winer KK, Yanovski JA, et al. Mutations in the $\mathrm{Ca}(2+)$-sensing receptor gene cause autosomal dominant and sporadic hypoparathyroidism. Hum Mol Genet 1996;5:601-6. 
[40] Hannan FM, Nesbit MA, Zhang C, et al. Identification of 70 calcium-sensing receptor mutations in hyper- and hypocalcaemic patients: evidence for clustering of extracellular domain mutations at calcium-binding sites. Hum Mol Genet 2012;21:2768-78.

[41] Mannstadt M, Harris M, Bravenboer B, et al. Germline mutations affecting G $\alpha 11$ in hypoparathyroidism. N Engl J Med 2013;368:2532-4.

[42] Nesbit MA, Hannan FM, Howles SA, et al. Mutations affecting G-protein subunit $\alpha 11$ in hypercalcemia and hypocalcemia. N Engl J Med 2013;368:2476-86.

[43] Roszko KL, Bi RD, Mannstadt M. Autosomal dominant hypocalcemia (hypoparathyroidism) types 1 and 2. Front Physiol 2016;7:458.

[44] Hannan FM, Olesen MK, Thakker RV. Calcimimetic and calcilytic therapies for inherited disorders of the calcium-sensing receptor signalling pathway. Br J Pharmacol 2017. https://doi.org/10.1111/bph.14086.

[45] McDonald-McGinn DM, Sullivan KE, Marino B, et al. 22q11.2 deletion syndrome. Nat Rev Dis Prim 2015;1:15071.

[46] Schuffenhauer S, Lichtner P, Peykar-Derakhshandeh P, et al. Deletion mapping on chromosome 10p and definition of a critical region for the second Di George syndrome locus (DGS2). Eur J Hum Genet 1998;6:213-25.

[47] Yagi H, Furutani Y, Hamada H, et al. Role of TBX1 in human del22q11.2 syndrome. Lancet 2003;362:1366-73.

[48] de la Morena MT, Eitson JL, Dozmorov IM, et al. Signature microRNA expression patterns identified in humans with 22q11.2 deletion/DiGeorge syndrome. Clin Immunol 2013;147:11-22.

[49] Kobrynski LJ, Sullivan KE. Velocardiofacial syndrome, DiGeorge syndrome: the chromosome 22q11.2 deletion syndromes. Lancet 2007;370:1443-52.

[50] Kruszka P, Addissie YA, McGinn DE, et al. 22q11.2 deletion syndrome in diverse populations. Am J Med Genet Part A 2017; 173:879-88.

[51] Palmer LD, Butcher NJ, Boot E, et al. Elucidating the diagnostic odyssey of 22q11.2 deletion syndrome. Am J Med Genet Part A 2018;176:936-44.

[52] Hsu P, Ma A, Wilson M, et al. CHARGE syndrome: a review. J Paediatr Child Health 2014;50:504-11.

[53] Ufartes R, Schwenty-Lara J, Freese L, et al. Sema3a plays a role in the pathogenesis of CHARGE syndrome. Hum Mol Genet 2018;27:1343-52.

[54] Grigorieva IV, Mirczuk S, Gaynor KU, et al. Gata3-deficient mice develop parathyroid abnormalities due to dysregulation of the parathyroid-specific transcription factor Gcm2. J Clin Invest 2010;120:2144-55.

[55] Van Esch H, Groenen P, Nesbit MA, et al. GATA3 haplo-insufficiency causes human HDR syndrome. Nature 2000;406: 419-22.

[56] Nesbit MA, Bowl MR, Harding B, et al. Characterization of GATA3 mutations in the hypoparathyroidism, deafness, and renal dysplasia (HDR) syndrome. J Biol Chem 2004;279:22624-34.

[57] Belge H, Dahan K, Cambier JF, et al. Clinical and mutational spectrum of hypoparathyroidism, deafness and renal dysplasia syndrome. Nephrol Dial Transplant 2017;32:830-7.

[58] Parvari R, Hershkovitz E, Grossman N, et al. HRD/Autosomal recessive Kenny-Caffey syndrome consortium: mutation of TBCE causes hypoparathyroidism-retardation-dysmorphism and autosomal recessive Kenny-Caffey syndrome. Nat Genet 2002;32:448-52.

[59] Padidela R, Kelberman D, Press M, et al. Mutation in the TBCE gene is associated with hypoparathyroidism-retardationdysmorphism syndrome featuring pituitary hormone deficiencies and hypoplasia of the anterior pituitary and the corpus callosum. J Clin Endocrinol Metab 2009;94:2686-91.

[60] Unger S, Górna MW, Le Béchec A, et al. FAM111A mutations result in hypoparathyroidism and impaired skeletal development. Am J Hum Genet 2013;92:990-5.

[61] Isojima T, Doi K, Mitsui J, et al. A recurrent de novo FAM111A mutation causes Kenny-Caffey syndrome type 2. J Bone Miner Res 2014;29:992-8.

*[62] Chow J, Rahman J, Achermann JC, et al. Mitochondrial disease and endocrine dysfunction. Nat Rev Endocrinol 2017;13: 92-104.

[63] Naiki M, Ochi N, Kato YS, et al. Mutations in HADHB, which encodes the $\beta$-subunit of mitochondrial trifunctional protein, cause infantile onset hypoparathyroidism and peripheral polyneuropathy. Am J Med Genet Part A 2014;164A:1180-7.

[64] Donoghue SE, Pitt JJ, Boneh A, et al. Smith-Lemli-Opitz syndrome: clinical and biochemical correlates. J Pediatr Endocrinol Metab 2018;31:451-9.

[65] Kisand K, Peterson P. Autoimmune polyendocrinopathy candidiasis ectodermal dystrophy. J Clin Immunol 2015;35: 463-78.

[66] Conteduca G, Indiveri F, Filaci G, et al. Beyond APECED: an update on the role of the autoimmune regulator gene (AIRE) in physiology and disease. Autoimmun Rev 2018;17:325-30.

[67] Meyer S, Woodward M, Hertel C, et al. APECED patient collaborative, Krohn K, Ranki A, Peterson P, Kisand K \& Hayday A. AIRE-deficient patients harbor unique high-affinity disease-ameliorating autoantibodies. Cell 2016;166:582-95.

[68] Alimohammadi M, Björklund P, Hallgren A, et al. Autoimmune polyendocrine syndrome type 1 and NALP5, a parathyroid autoantigen. N Engl J Med 2008;358:1018-28.

[69] Kemp EH, Habibullah M, Kluger N, et al. Prevalence and clinical associations of calcium-sensing receptor and NALP5 autoantibodies in Finnish APECED patients. J Clin Endocrinol Metab 2014;99:1064-71.

[70] Ferre EM, Rose SR, Rosenzweig SD, et al. Redefined clinical features and diagnostic criteria in autoimmune polyendocrinopathy-candidiasis-ectodermal dystrophy. J Clin Investig Insight 2016;1. pii: e88782.

[71] Weetman AP. Auto-immune hypoparathyroidism. Ann Endocrinol (Paris) 2015;76:89-91.

*[72] Stack Jr BC, Bimston DN, Bodenner DL, et al. American Association of Clinical Endocrinologists and American College of Endocrinology Disease state clinical review: postoperative hypoparathyroidism-definitions and management. Endocr Pract 2015;21:674-85.

[73] Orloff LA, Wiseman SM, Bernet VJ, et al. American thyroid association statement on postoperative hypoparathyroidism: diagnosis, Prevention, and management in adults. Thyroid 2018 Jun 29. https://doi.org/10.1089/thy.2017.0309.

[74] Asari R, Passler C, Kaczirek K, et al. Hypoparathyroidism after total thyroidectomy: a prospective study. Arch Surg 2008; $143: 132-7$. 
[75] Nordenström E, Bergenfelz A, Almquist M. Permanent hypoparathyroidism after total thyroidectomy in children: results from a national registry. World J Surg 2018. https://doi.org/10.1007/s00268-018-4552-7.

[76] Edafe O, Antakia R, Laskar N, et al. Systematic review and meta-analysis of predictors of post-thyroidectomy hypocalcaemia. Br J Surg 2014;101:307-20.

[77] Bentrem DJ, Rademaker A, Angelos P. Evaluation of serum calcium levels in predicting hypoparathyroidism after total/ near-total thyroidectomy or parathyroidectomy. Am Surg 2001;67:249-51.

[78] Cho JN, Park WS, Min SY. Predictors and risk factors of hypoparathyroidism after total thyroidectomy. Int J Surg 2016;34: 47-52. 